\title{
Associating Factors Regarding Nasogastric Tube Removal in Patients With Dysphagia After Stroke
}

\author{
Jong Hwa Lee, MD, Sang Beom Kim, MD, Kyeong Woo Lee, MD, \\ Sook Joung Lee, MD, Jin Gee Park, MD, Jae Won Ri, MD \\ Department of Physical Medicine and Rehabilitation and Regional Cardiocerebrovascular Center, \\ Dong-A University Hospital, Busan, Korea
}

Objective To demonstrate associating factors regarding nasogastric tube (NGT) removal in patients with dysphagia after stroke.

Methods This study is a retrospective medical chart review. Patients were divided into non-brain stem (NBS) and brain stem (BS) groups. A videofluoroscopic swallowing study was conducted until swallowing functions were recovered. Initial disease status was measured using the National Institutes of Health Stroke Scale (NIHSS) and the modified Rankin Scale (mRS). Risk factors related to stroke were evaluated. The penetration-aspiration scale (PAS) was used as the swallowing test. Functional status was measured by Mini-Mental Status Examination (MMSE) and Modified Barthel Index (MBI). Within each group, initial evaluations and their subsequent changes were compared according to the NGT removal status. Correlation between the NGT removal time and other initial factors were evaluated.

Results Ninety-nine patients were allocated to the NBS group and 39 to the BS group. In NBS, age, PAS, MMSE, and MBI were significantly different according to the NGT removal status. In BS, smoking and PAS were significantly different. In NBS, changes in PAS, MMSE, and MBI were significantly different according to the NGT removal status. In BS, only PAS change was significantly different. In NBS, initial NIHSS, mRS, MMSE, and MBI were correlated with removal time.

Conclusion In stroke patients with NTG, younger age, better initial disease and functional status seems to remove NGT in NBS stroke. Therefore, when deciding to remove NGT, those three factors should be considered discreetly.

Keywords Stroke, Dysphagia, Nasogastric tube, Fluoroscopy

Received June 25, 2013; Accepted September 13, 2013

Corresponding author: Jin Gee Park

Department of Physical Medicine and Rehabilitation, Dong-A University Hospital, 26 Daesingongwon-ro, Seo-gu, Busan 602-715, Korea

Tel: +82-51-240-5690, Fax: +82-51-254-8511, E-mail: jingee00@naver.com

@ This is an open-access article distributed under the terms of the Creative Commons Attribution Non-Commercial License (http://creativecommons. org/licenses/by-nc/3.0) which permits unrestricted noncommercial use, distribution, and reproduction in any medium, provided the original work is properly cited.

Copyright $\odot 2014$ by Korean Academy of Rehabilitation Medicine

\section{INTRODUCTION}

Stroke is one of the common causes of dysphagia. Several studies have reported that $30 \%-50 \%$ of stroke patients exhibit dysphagia [1-5]. Those with severe dysphagia, especially due to acute stroke, nasogastric tube (NGT) is usually recommended as a safe way of supplying nutrition. Usually, NGT-fed patients are evaluated by the videofluoroscopic swallowing study (VFSS) periodically to decide their feeding material. According to the test 
results, they could have the tube removed or maintain it. However, after discharging from the hospital, most of the patients do not keep up with the test periodically and having NGT remaining in the body. Several studies have shown that persistent dysphagia is closely associated with poor outcome, including lower functional ability and increased mortality [6-10]. Also, prolonged NGT insertion can lead to complications, such as lesions to the nasal wing, chronic sinusitis, gastro-esophageal reflux, and aspiration pneumonia [11]. These complications can worsen the patient's outcome. Therefore, appropriate removal of NGT in stroke patient with dysphagia is critically important.

Several studies have reported the effect of NGT insertion on swallowing function in patients with stroke $[12,13]$. However, few studies have reported regarding NGT removal in patients with stroke. Thus, our purpose of this study was to demonstrate associating factors regarding NGT removal in patients with dysphagia after stroke.

\section{MATERIALS AND METHODS}

Data from stroke patients who were admitted to our Regional Cardiocerebrovascular Center from March 2009 to February 2012 were retrospectively extracted from their medical records. Patients were inserted NGT for the first time for the treatment of dysphagia after stroke. NGT insertion criteria were altered mental status, unable to open the mouth, and cough or wet voice after swallowing solid or fluid. To minimize variability arising from health status before stroke, inclusion was limited to patients who had just suffered their first-ever stroke and who were previously functionally independent in daily living. During the hospitalization, patients underwent physical therapy and occupational therapy for 30 minutes, twice a day, 5 times a week, as well as swallowing therapy for 20 minutes, twice a week. Patients with hemorrhagic stroke were excluded from this study.

Patients were divided into two groups according to their lesion site. Non-brain stem (NBS) group had lesions located in the middle, anterior or posterior cerebral artery territory. Brain stem (BS) group had lesions located in the midbrain, medulla or pons. They all underwent VFSS within 5 days after stroke onset and followed up every 2 weeks until their swallowing function recovered or were discharged from the hospital.

Initial disease status was measured using the National Institutes of Health Stroke Scale (NIHSS) and modified Rankin Scale (mRS). Risk factors related to stroke were evaluated in all patients. Risk factors were hypertension (HTN), diabetes mellitus (DM), alcohol, smoking, and atrial fibrillation (AF). The penetration-aspiration scale (PAS) was used as the swallowing test. Functional status was measured by Mini-Mental Status Examination (MMSE) and Modified Barthel Index (MBI). All the evaluations were taken at the initial state, a few days after the removal of NGT in those who could have it removed and few days after the last VFSS follow-up in those who could not have their NGT removed. Within each group, initial evaluations and factors were compared according to the NGT removal status. Changes were compared according to the NGT removal status. Correlation between NGT removal time and age, initial NIHSS, mRS, PAS, MMSE, and MBI were evaluated in patients who could remove NGT.

Statistical analysis was performed using SPSS ver. 12.0 for Windows (SPSS Inc., Chicago, IL, USA). The Mann Whitney U test was used to compare age, NIHSS, mRS, PAS, MMSE, and MBI according to the NGT removal status. The chi-square test was used to compare HTN, DM, alcohol, smoking, and AF according to the NGT removal status. Spearman correlation was used to evaluate the relation between removal time and age, NHISS, mRS, PAS, MMSE, and MBI. The p-values less than 0.05 were con-

Table 1. Demographic characteristics of the two study groups

\begin{tabular}{lcc}
\hline \multicolumn{1}{c}{ Characteristic } & NBS $(\mathbf{n}=\mathbf{9 9})$ & BS $(\mathbf{n}=\mathbf{3 9})$ \\
\hline Sex (male:female) & $72: 27$ & $34: 5$ \\
\hline Age (yr) & $69.3 \pm 9.7$ & $62.8 \pm 12.8$ \\
Lesion & & \\
MCA & $91(91.9)$ & \\
ACA & $5(5.0)$ & \\
PCA & $3(3.0)$ & \\
Midbrain & & $5(12.8)$ \\
Pons & & $10(25.6)$ \\
Medulla & & $24(61.5)$ \\
\hline
\end{tabular}

Values are presented as mean \pm standard deviation or number (\%).

NBS, non-brain stem; BS, brain stem; MCA, middle cerebral artery; ACA, anterior cerebral artery; PCA, posterior cerebral artery. 
sidered statistically significant.

\section{RESULTS}

Ninety-nine patients were in the NBS group and 39 patients in the BS group. The mean age was $69.3 \pm 9.7$ years in the NBS group and $62.8 \pm 12.8$ years in the BS group. Middle cerebral artery was the most common lesion in the NBS group and medulla in the BS group. Other demographic characteristics are listed in Table 1.

Initial evaluations and subsequent changes are listed in Table 2. The mean removal time was $25.2 \pm 19.3$ days in the NBS group and $16.4 \pm 14.2$ days in the BS group. In the NBS group, $58 \%$ of the patients eventually had the tube removed and in the BS group, $44 \%$ did. In the NBS group, the mean age was $72.8 \pm 7.6$ years in patient who could not have NGT removed and $66.8 \pm 10.3$ years in those who could have it removed. The mean PAS was $7.8 \pm 0.5$ in patients who could not have NGT removed and 7.0 \pm 0.8 in patient who have it removed. The mean MMSE was 6.6 \pm 7.5 in patients who could not have NGT removed and $11.8 \pm 8.1$ in patient who could have it removed. The mean MBI was $25.0 \pm 17.9$ in patients who could not have NGT removed and $35.4 \pm 24.1$ in patient who could have it removed. These factors were statistically significant $(\mathrm{p}<0.05)$. However, NIHSS, mRS, HTN, DM, alcohol, smoking, and AF were not statistically significant. In the BS group, $50.0 \%$ of patients who could not have NGT removed were smokers and $17.6 \%$ of patients who could have it removed were smokers. The mean PAS was $7.9 \pm 0.3$

Table 2. Initial evaluations and subsequent changes

\begin{tabular}{|c|c|c|c|c|}
\hline \multirow{2}{*}{ Factor } & \multicolumn{2}{|c|}{ NBS } & \multicolumn{2}{|c|}{ BS } \\
\hline & Not remove $(n=42)$ & Remove $(n=57)$ & Not remove $(n=22)$ & Remove $(n=17)$ \\
\hline Removal time (day) & - & $25.2 \pm 19.3$ & - & $16.4 \pm 14.2$ \\
\hline Follow-up time (day) & $46.3 \pm 28.0$ & & $52.1 \pm 30.4$ & \\
\hline Age (yr) & $72.8 \pm 7.6$ & $66.8 \pm 10.3^{*}$ & $60.6 \pm 14.0$ & $65.7 \pm 10.9$ \\
\hline \multicolumn{5}{|l|}{ Disease status } \\
\hline NIHSS & $14.0 \pm 4.4$ & $12.8 \pm 6.1$ & $7.4 \pm 6.3$ & $5.2 \pm 3.5$ \\
\hline$\Delta$ NIHSS & $-2.7 \pm 2.4$ & $-2.6 \pm 2.2$ & $-0.6 \pm 0.7$ & $-0.7 \pm 1.0$ \\
\hline mRS & $4.3 \pm 0.8$ & $4.2 \pm 0.8$ & $3.4 \pm 1.0$ & $2.8 \pm 1.1$ \\
\hline$\Delta \mathrm{mRS}$ & $-0.3 \pm 0.5$ & $-0.5 \pm 0.5$ & $-0.2 \pm 0.4$ & $-0.2 \pm 0.4$ \\
\hline \multicolumn{5}{|l|}{ Risk factors } \\
\hline HTN & $25(59.5)$ & $35(61.4)$ & $13(59.0)$ & $12(70.6)$ \\
\hline $\mathrm{DM}$ & $7(16.7)$ & $17(29.8)$ & $7(31.8)$ & $6(35.3)$ \\
\hline Alcohol & $10(23.8)$ & $22(38.6)$ & $12(54.5)$ & $5(29.4)$ \\
\hline Smoking & $11(26.2)$ & $23(40.4)$ & $11(50.0)$ & $3(17.6)^{*}$ \\
\hline $\mathrm{AF}$ & $12(28.6)$ & $22(38.6)$ & $1(4.5)$ & $4(23.5)$ \\
\hline \multicolumn{5}{|l|}{ Swallowing test } \\
\hline PAS & $7.8 \pm 0.5$ & $7.0 \pm 0.8^{*}$ & $7.9 \pm 0.3$ & $6.2 \pm 0.4^{*}$ \\
\hline$\triangle \mathrm{PAS}$ & $-0.3 \pm 0.5$ & $-3.5 \pm 0.9 *$ & $-0.5 \pm 0.5$ & $-1.8 \pm 0.8^{*}$ \\
\hline \multicolumn{5}{|l|}{ Functional status } \\
\hline MMSE & $6.6 \pm 7.5$ & $11.8 \pm 8.1^{*}$ & $21.6 \pm 7.4$ & $21.2 \pm 7.4$ \\
\hline$\triangle \mathrm{MMSE}$ & $2.0 \pm 3.3$ & $2.9 \pm 2.4^{*}$ & $1.3 \pm 1.5$ & $1.7 \pm 1.3$ \\
\hline MBI & $25.0 \pm 17.9$ & $35.4 \pm 24.1^{*}$ & $48.0 \pm 24.1$ & $59.4 \pm 14.5$ \\
\hline$\Delta \mathrm{MBI}$ & $7.0 \pm 6.1$ & $9.8 \pm 7.5^{*}$ & $11.1 \pm 5.9$ & $13.1 \pm 6.3$ \\
\hline
\end{tabular}

Values are presented as mean \pm standard deviation or number (\%).

NBS, non-brain stem; BS, brain stem; NIHSS, National Institutes of Health Stroke Scale; mRS, modified Rankin Scale; HTN, hypertension; DM, diabetes mellitus; AF, atrial fibrillation; PAS, penetration-aspiration scale; MMSE, MiniMental Status Examination; MBI, Modified Barthel Index.

${ }^{*} \mathrm{p}<0.05$ by Mann Whitney $\mathrm{U}$ test and chi-square test between not remove and remove. 
Table 3. Correlation between the removal time of nasogastric tube and other initial factors

\begin{tabular}{lcccc}
\hline & NBS coefficient & p-value & BS coefficient & p-value \\
\hline Age & -0.203 & 0.065 & 0.044 & 0.433 \\
NIHSS & 0.317 & $0.008^{* *}$ & 0.281 & 0.137 \\
mRS & 0.349 & $0.004^{* *}$ & 0.222 & 0.196 \\
PAS & -0.019 & 0.444 & 0.028 & 0.457 \\
MMSE & -0.286 & $0.016^{*}$ & -0.047 & 0.429 \\
MBI & -0.289 & $0.014^{*}$ & -0.134 & 0.304 \\
\hline
\end{tabular}

NBS, non-brain stem; BS, brain stem; NIHSS, National Institutes of Health Stroke Scale; mRS, modified Rankin Scale; PAS, penetration-aspiration scale; MMSE, Mini-Mental Status Examination; MBI, Modified Barthel Index. ${ }^{*} \mathrm{p}<0.05,{ }^{* *} \mathrm{p}<0.01$ by Spearman correlation.

(A)

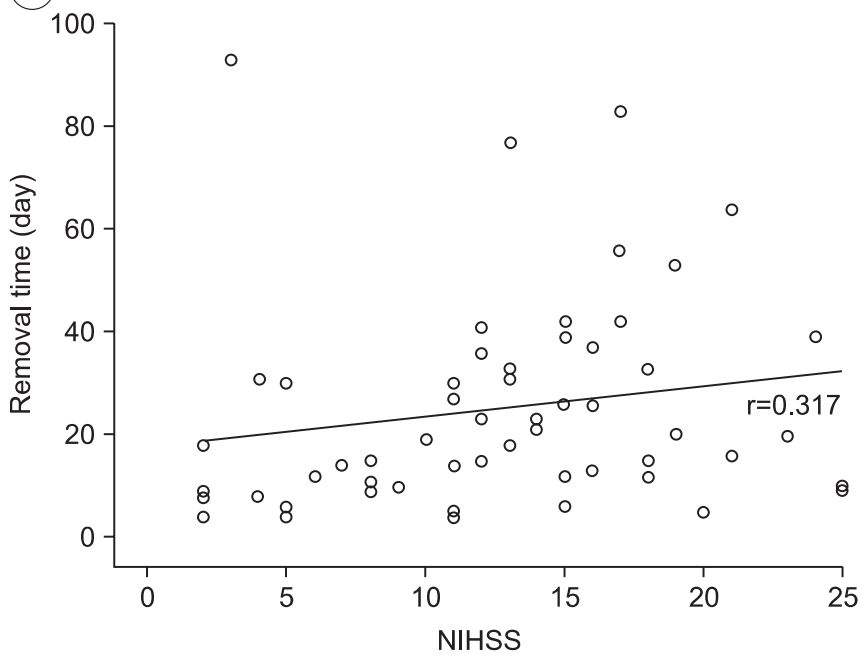

(C)

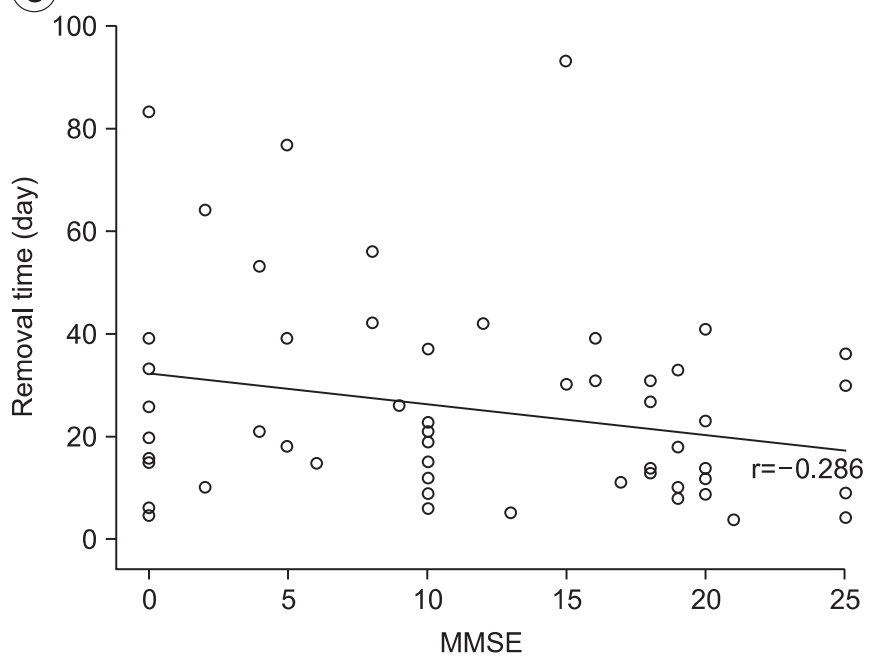

(B)

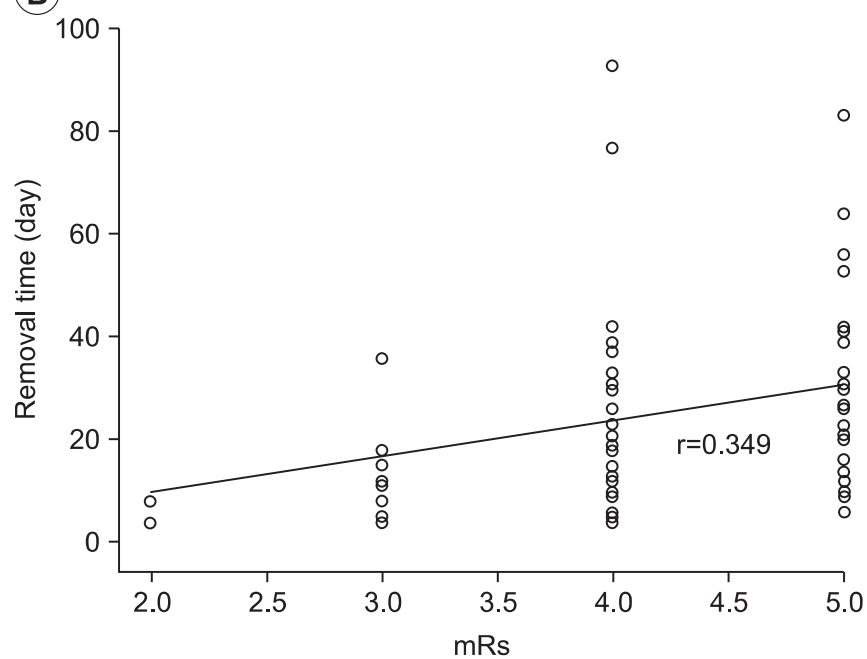

(D)

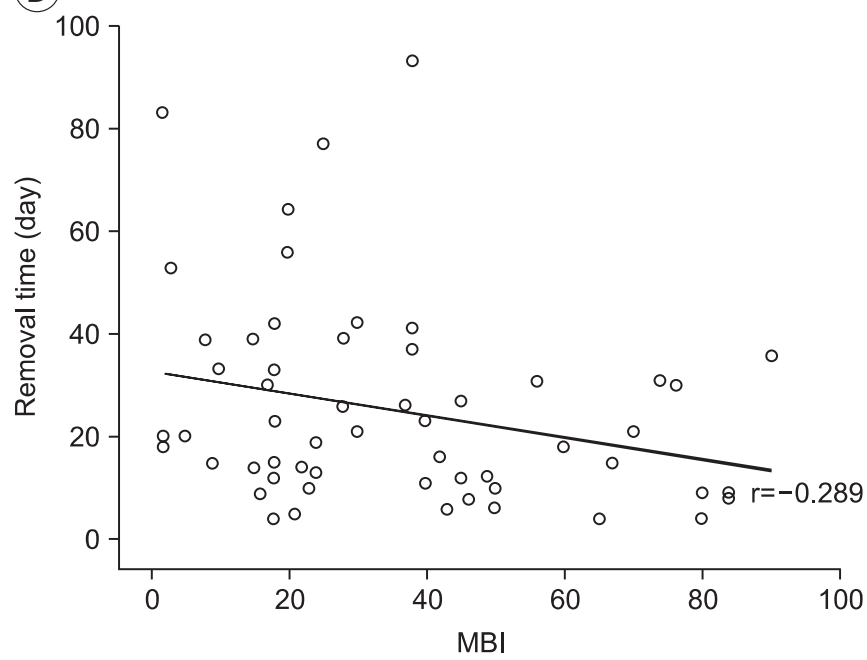

Fig. 1. In non-brain stem stroke patients, National Institutes of Health Stroke Scale (NIHSS, A) and modified Rankin Scale (mRS, B) showed a positive correlation, Mini-Mental Status Examination (MMSE, C) and Modified Barthel Index (MBI, D) showed a negative correlation with removal time. 
in patients who could not have NGT removed and 6.2 \pm 0.4 in patients who could have it removed. Both factors were statistically significant ( $\mathrm{p}<0.05)$. Age, NIHSS, mRS, HTN, DM, alcohol, AF, MMSE, and MBI were not statistically significant.

In the NBS group, the change in mean PAS was $-0.3 \pm 0.5$ in patients who could have NGT removed and $-3.5 \pm 0.9$ in patients who could have it removed. The change in mean MMSE was 2.0 \pm 3.3 in patients who could not have NGT removed and $2.9 \pm 2.4$ in patients who could have it removed. The change in mean MBI was 7.0 6 .1 in patients who could have NGT removed and $9.8 \pm 7.5$ in patients who could have it removed. These factors were statistically significant $(\mathrm{p}<0.05)$. In the $B S$ group, the change in mean PAS was $-0.5 \pm 0.5$ in patients who could not have NGT removed and $-1.8 \pm 0.8$ in patients who could have it removed. Change in PAS was the only statistically significant factor $(\mathrm{p}<0.05)$.

Correlation between the removal time of NGT and other initial factors are listed in Table 3. In the NBS group, NIHSS ( $\mathrm{r}=0.317, \mathrm{p}=0.008$ ) (Fig. $1 \mathrm{~A}$ ) and $\mathrm{mRS}(\mathrm{r}=0.349$, $\mathrm{p}=0.004$ ) (Fig. 1B) showed a positive correlation; MMSE $(\mathrm{r}=-0.286, \mathrm{p}=0.016)$ (Fig. 1C) and MBI $(\mathrm{r}=-0.289, \mathrm{p}=0.014)$ (Fig. 1D) showed a negative correlation with removal time. In the BS group, none of the factors showed correlation.

\section{DISCUSSION}

The purpose of the present study was to demonstrate associating factors regarding NGT removal in patients with dysphagia after stroke. In the NBS group, age, initial PAS, MMSE, and MBI were significantly different between not removed and removed patients. In the BS group, smoking and initial PAS were significantly different. In the NBS group, changes in PAS, MMSE, and MBI were significantly different between not removed and removed patients. In the BS group, change in PAS was the only significantly different factor. In correlation test, initial NIHSS, mRS, MMSE, and MBI showed significance in the NBS group. However, no significance was found in the BS group.

In the NBS group, stroke patients with younger age, higher MMSE and MBI showed a better chance of NGT removal. These findings were concordant with other studies, in which a greater number of swallowing disor- ders was found at the age of 65 to 75 years $[14,15]$. Another study reported that age, Functional Independence Measure motor and cognition scores and days after stroke onset can readily predict oral feeding outcome [16]. Ickenstein et al. [17] reported that functional measurements including cognitive function could play an important role in swallowing regeneration in stroke patients. Several studies assume that the older the age, the greater the chances of the aging body to result in phonoarticulatoty, which would undermine the function of the stomatognathic system and subsequently cause swallowing disorders, especially after stroke $[14,15,18]$. The results of these reports imply that age and functional status are highly associated with dysphagia and which may lead to usage of NGT in stroke patients. The reason for the association with higher cognitive function and NGT removal is that higher cognition makes it possible for the patient to learn and use compensatory measures for swallowing.

Even though the NBS group had some associating factors, the BS group did not have any associating factors except for smoking. There was a report that in stroke patients, risk factors were HTN, DM, and smoking followed by frequency. Smoking was in the third place. But only DM was predictor for swallowing disorders [19]. The study showed no concordance with our study. Thus, a further study might be needed. Impaired endogenous fibrinolysis and reduced blood flow to the brain secondary to smoking-induced vasoconstriction may contribute to stroke in small vessels [20].

In other reports, symptoms of dysphagia in stroke patients were different regarding the site of the lesion. Cortical lesions may give rise to difficulty in controlling the voluntary movement of chewing, thus impairing transportation of the food bolus during the oral and pharyngeal phase of swallowing and increase the chances of tracheal aspiration. BS lesions are less frequent, but result in severe impairment of swallowing because of the location of most of the nuclei of the cranial nerves [7,21]. Teismann et al. [22] reported that hemispheric and BS stroke patients with dysphagia showed different swallowing processes in the brain. Similar to these reports, our study could find different patterns of swallowing between the two groups. The BS group patients had lower NIHSS and mRS, and higher PAS, MMSE and MBI than the NBS group. This implies that stroke in the BS has relatively better disease status and function but more severely im- 
paired swallowing.

Previous studies have reported regarding the relation between function and dysphagia in stroke patients. Okubo et al. [23] showed that NIHSS is highly sensitive and specific in detecting dysphagia. Kumar et al. [24] reported that baseline NIHSS could predict percutaneous endoscopic gastrostomy placement during hospitalization from an acute-subacute hemispheric infarction in patients with severe dysphagia. Also, there was a study reporting that high indexes on mRS and low indexes on the MBI could be risk factors for swallowing dysfunction after stroke [25]. The present study also showed some correlations in the NBS group. Higher the NIHSS and mRS, and lower the MMSE and MBI showed the more delay in the removal time of NGT. In particular, initial disease status showed a strong correlation. Thus, it can be deduced initial stroke status plays an important role in NGT removal. However, no correlation was found in the BS group. The reason could be the small sample size of the BS group.

Several studies showed that recovery of dysphagia occurs over the first few weeks after the event, and dysphagia remains in a small number of patients $[14,18]$. In our study, the follow-up period was not long enough. The mean follow-up time was $46.3 \pm 28.0$ days in the NBS group and 52.1 \pm 30.4 days in the BS group. The stroke patients' maximum inpatient rehabilitation duration was about 8 weeks or less. Therefore, a considerable number of patients could not obtain full recovery of dysphagia. And discharged hospital with NGT remained in the body. If patients could have follow-up VFSS, the removal rate would be different.

The strength of our study was that even though there have been some reports on the effect of NGT insertion on swallowing function, none of the studies have evaluated removal of NGT in patients with stroke. As a result, we were able to discover factors affecting the NGT removal time. However, the present study has some limitations. First, BS group sample size was relatively small compared to the NBS group to find out conclusive factors. Second, there were no patients with hemorrhagic stroke. Third, VFSS follow-up was not long enough for patients to achieve full recovery in swallowing. Thus, the results of the study may be applicable only for acute and subacute stroke patients.

In stroke patients with NGT, relatively young and better functional status patients could have the NGT removed in NBS stroke. Also, better initial disease and functional status were correlated with removal time in NBS stroke. When making a decision to remove NGT in stroke patients, it is important to consider patient's age, initial disease status and initial functions discreetly.

\section{CONFLICT OF INTEREST}

No potential conflict of interest relevant to this article was reported.

\section{REFERENCES}

1. Gordon C, Hewer RL, Wade DT. Dysphagia in acute stroke. Br Med J (Clin Res Ed) 1987;295:411-4.

2. Horner J, Massey EW, Riski JE, Lathrop DL, Chase KN. Aspiration following stroke: clinical correlates and outcome. Neurology 1988;38:1359-62.

3. Barer DH. The natural history and functional consequences of dysphagia after hemispheric stroke. J Neurol Neurosurg Psychiatry 1989;52:236-41.

4. Horner J, Buoyer FG, Alberts MJ, Helms MJ. Dysphagia following brain-stem stroke: clinical correlates and outcome. Arch Neurol 1991;48:1170-3.

5. Mann G, Hankey GJ, Cameron D. Swallowing disorders following acute stroke: prevalence and diagnostic accuracy. Cerebrovasc Dis 2000;10:380-6.

6. Smithard DG, O'Neill PA, Parks C, Morris J. Complications and outcome after acute stroke: does dysphagia matter? Stroke 1996;27:1200-4.

7. Paciaroni M, Mazzotta G, Corea F, Caso V, Venti M, Milia P, et al. Dysphagia following stroke. Eur Neurol 2004;51:162-7.

8. Kalra L, Smith DH, Crome P. Stroke in patients aged over 75 years: outcome and predictors. Postgrad Med J 1993;69:33-6.

9. Schmidt J, Holas M, Halvorson K, Reding M. Videofluoroscopic evidence of aspiration predicts pneumonia and death but not dehydration following stroke. Dysphagia 1994;9:7-11.

10. Smithard DG, Smeeton NC, Wolfe CD. Long-term outcome after stroke: does dysphagia matter? Age Ageing 2007;36:90-4.

11. Gomes CA Jr, Lustosa SA, Matos D, Andriolo RB, Waisberg DR, Waisberg J. Percutaneous endoscopic gastrostomy versus nasogastric tube feeding for adults 
with swallowing disturbances. Cochrane Database Syst Rev 2012;3:CD008096.

12. Dennis MS, Lewis SC, Warlow C; FOOD Trial Collaboration. Effect of timing and method of enteral tube feeding for dysphagic stroke patients (FOOD): a multicentre randomised controlled trial. Lancet 2005;365:764-72.

13. Wang TG, Wu MC, Chang YC, Hsiao TY, Lien IN. The effect of nasogastric tubes on swallowing function in persons with dysphagia following stroke. Arch Phys Med Rehabil 2006;87:1270-3.

14. Mann G, Hankey GJ, Cameron D. Swallowing function after stroke: prognosis and prognostic factors at 6 months. Stroke 1999;30:744-8.

15. Ros L, Garcia M, Prat J, Gonzalez C, Gimeno C, Albert A, et al. Predictors of nosocomial infection in acute stroke: relation with morbimortality and outcome. Med Clin (Barc) 2007;128:441-7.

16. Oto T, Kandori Y, Ohta T, Domen K, Koyama T. Predicting the chance of weaning dysphagic stroke patients from enteral nutrition: a multivariate logistic modelling study. Eur J Phys Rehabil Med 2009;45:35562.

17. Ickenstein GW, Stein J, Ambrosi D, Goldstein R, Horn M, Bogdahn U. Predictors of survival after severe dysphagic stroke. J Neurol 2005;252:1510-6.

18. Mann G, Hankey GJ. Initial clinical and demographic predictors of swallowing impairment following acute stroke. Dysphagia 2001;16:208-15.

19. Remesso GC, Fukujima MM, Chiappetta AL, Oda AL, Aguiar AS, Oliveira Ade S, et al. Swallowing disorders after ischemic stroke. Arq Neuropsiquiatr 2011;69:7859.

20. Shah RS, Cole JW. Smoking and stroke: the more you smoke the more you stroke. Expert Rev Cardiovasc Ther 2010;8:917-32.

21. Martino R, Foley N, Bhogal S, Diamant N, Speechley M, Teasell R. Dysphagia after stroke: incidence, diagnosis, and pulmonary complications. Stroke 2005;36:2756-63.

22. Teismann IK, Suntrup S, Warnecke T, Steinstrater O, Fischer M, Floel A, et al. Cortical swallowing processing in early subacute stroke. BMC Neurol 2011;11:34.

23. Okubo PC, Fabio SR, Domenis DR, Takayanagui OM. Using the National Institute of Health Stroke Scale to predict dysphagia in acute ischemic stroke. Cerebrovasc Dis 2012;33:501-7.

24. Kumar S, Langmore S, Goddeau RP Jr, Alhazzani A, Selim M, Caplan LR, et al. Predictors of percutaneous endoscopic gastrostomy tube placement in patients with severe dysphagia from an acute-subacute hemispheric infarction. J Stroke Cerebrovasc Dis 2012;21:114-20.

25. Baroni AF, Fabio SR, Dantas RO. Risk factors for swallowing dysfunction in stroke patients. Arq Gastroenterol 2012;49:118-24. 